PROCEEDINGS OF THE

AMERICAN MATHEMATICAL SOCIETY

Volume 125, Number 9, September 1997, Pages 2777-2778

S 0002-9939(97)04114-2

\title{
CONSTRUCTION OF ANR TOPOLOGIES ON CERTAIN GROUPS
}

\author{
BERND GÜNTHER
}

(Communicated by Thomas Goodwillie)

\begin{abstract}
A method is shown to construct ANR-topologies on topological groups of suitable homotopy type.
\end{abstract}

It is well known that every simple countable CW-complex with vanishing Postnikov invariants has the homotopy type of a topological Abelian group $G$, that can be taken as realization of a countable simplicial Abelian group and hence is a countable CW-complex [5, Ch.V]. In particular this means that the homotopy groups $\pi_{n}(G)$ can be chosen as an arbitrarily prescribed sequence of countable Abelian groups. However, there are situations where it would be preferable to have $G$ as an ANR-space instead of a CW-complex. One would guess that a suitable topology exists on $G$, because by subdivision it can be turned into a polyhedron and then can get the metric topology, but the subdivision process necessarily leaves the range of simplicial Abelian groups and need not produce a group topology. Instead, we follow a method developed by Cauty in [1].

Theorem. Let $G$ be a locally contractible, not necessarily Abelian group, every open subset of which is $\sigma$-compact, such that $G$ is the union of a sequence of finite dimensional compact metric spaces and has the homotopy type of a $C W$-complex. Then $G$ carries a metrizable group topology, coarser than the original one but of the same homotopy type, which turns $G$ into an ANR-space.

All these requirements are satisfied, for instance, if $G$ itself is a countable $\mathrm{CW}$ complex.

Proof. We construct a decreasing sequence of open neighborhoods $V_{n}$ of the identity $1 \in G$ such that

1. $\bigcap_{n=1}^{\infty} V_{n}=\{1\}$,

2. the inclusion map $V_{n+1} \hookrightarrow V_{n}$ is null homotopic,

3. if $H_{m}: V_{m+1} \times I \rightarrow V_{m}$ is the null homotopy from 2 , then for any $g \in V_{m+1}$ and each $n \geq m$ there exist $N \geq n$ and $\varepsilon>0$ such that $g^{\prime} \in g V_{N},\left|t^{\prime}-t\right|<$ $\varepsilon \Rightarrow H_{m}\left(g^{\prime}, t^{\prime}\right) \in H_{m}(g, t) V_{n}$,

4. $V_{n}^{-1}=V_{n}$ and $V_{n+1} V_{n+1} \subseteq V_{n}$,

5. for each $n$ and every $g \in G$ there exists $N \geq n$ with $g^{-1} V_{N} g \subseteq V_{n}$.

Received by the editors April 1, 1996.

1991 Mathematics Subject Classification. Primary 54H11, 54C55, 22A05.

Key words and phrases. Homotopy type of topological groups, ANR-spaces, CW-complexes.

(C)1997 American Mathematical Society 
All of these properties are immediately clear with the exception of 3 and 5 . We construct $V_{n}$ inductively. Let $U_{n}$ be a sequence of neighborhoods of the identity with $\bigcap_{n=1}^{\infty} U_{n}=\{1\}$; it exists because the open set $G \backslash\{1\}$ is $\sigma$-compact and therefore $\{1\}$ is a $G_{\delta}$. Choose $V_{1}$ with $V_{1}=V_{1}^{-1} \subseteq U_{1}$, otherwise arbitrarily. Now suppose that $V_{1}, \ldots, V_{n}$ are already constructed such that $2,4, V_{k} \subseteq U_{k}$ and the following properties hold:

3a. For $k<m \leq n$ there exist a sequence of neighborhoods of the identity $\left\{W_{i}^{k m} \mid\right.$ $i \in \mathbb{N}\}$ and a sequence of numbers $\varepsilon_{i}^{k m}>0$ such that for each $g \in V_{k+1}$ there exists $i$ with $g^{\prime} \in g W_{i}^{k m},\left|t^{\prime}-t\right|<\varepsilon_{i}^{k m} \Rightarrow H_{k}\left(g^{\prime}, t^{\prime}\right) \in H_{k}(g, t) V_{m}$.

3b. $V_{n} \subseteq \bigcap_{k<m<n, i \leq n} W_{i}^{k m}$.

5a. For every $g \in K_{n}$ we have $g^{-1} V_{n+1} g \subseteq V_{n}$, where $K_{m}$ is an increasing sequence of compacta covering $G$.

To pass from $n$ to $n+1$ choose $V_{n+1}$ subject to conditions $2,3 \mathrm{~b}, 4,5 \mathrm{a}$ and $V_{n+1} \subseteq$ $V_{n} \cap U_{n+1}$. It now remains to construct the neighborhoods $W_{i}^{k, n+1}$ for $k \leq n$. By assumption we can find a sequence of compact sets $C_{i} \subseteq V_{k+1}$ with $\bigcup_{i=1}^{\infty} C_{i}=$ $V_{k+1}$. For each of them we choose a neighborhood of the identity $W_{i}^{k, n+1}$ with $C_{i} W_{i}^{k, n+1} \subseteq V_{k+1}$ and a number $\varepsilon_{i}^{k, n+1}>0$ such that $g \in C_{i}, g^{\prime} \in g W_{i}^{k, n+1}$ and $\left|t^{\prime}-t\right|<\varepsilon_{i}^{k, n+1}$ implies $H_{k}\left(g^{\prime}, t^{\prime}\right) \in H_{k}(g, t) V_{m}$. It is clear that the whole sequence of neighborhoods $V_{n}$ satisfies $1-5$ and hence defines a metrizable group topology on $G$, which is coarser than the original topology. Denote by $\tilde{G}$ the group $G$ topologized by our neighborhood system $V_{n}$. 3 ensures that the homotopies $H_{n}$ are continuous with respect to our metrizable topology, and hence that $\tilde{G}$ is locally contractible. It now follows from [3] that $\tilde{G}$ is an ANR-space and from [4, Theorem 1] that the identity map $G \rightarrow \tilde{G}$ is a homotopy equivalence.

In a similar way it can be proved that the construction is "almost" functorial in the sense that for any family of continuous homomorphisms $f_{\lambda}: G_{\lambda} \rightarrow H_{\lambda}$ between groups satisfying the assumptions of our theorem, such that each group appears at most countably often among the source groups $G_{\lambda}$ the ANR group topology can be chosen in such a way that all homomorphisms $f_{\lambda}: \tilde{G}_{\lambda} \rightarrow \tilde{H}_{\lambda}$ are continuous. The countability assumption seems to be essential.

\section{REFERENCES}

1. R. Cauty: Un espace métrique linéaire qui n'est pas un réracte absolu; Fund. Math. 146 (1994) 85-99. MR 95j:54022

2. A. Dold, R. Thom: Quasifaserungen und unendliche symmetrische Produkte; Ann. of Math. 67 (1958) 239-281. MR 20:3542

3. W.E. Haver: Locally contractible spaces that are absolute neighborhood retracts; Proc. Amer. Math. Soc. 40 (1973) 280-284. MR 48:9645

4. G. Kozlowski: Factorization of Certain Maps up to Homotopy; Proc. Amer. Math. Soc. 21 (1969) 88-92. MR 38:6588

5. J.P. May: Simplicial Objects in Algebraic Topology; University of Chicago Press, Midway Reprint (1992). MR 93m:55025

Fachbereich Mathematik, Johann Wolfgang Goethe-Universität, Robert-MayerStrasse 6-10, 60054 Frankfurt, Germany

E-mail address: guenther@mathematik.uni-frankfurt.d400.de 